Thomae, Nico; Plagwitz, Kai-Uwe; Husar, Peter; Henning, Günter:

Hough-Transformation zur Bildverarbeitung bei der Blickrichtungsbestimmung

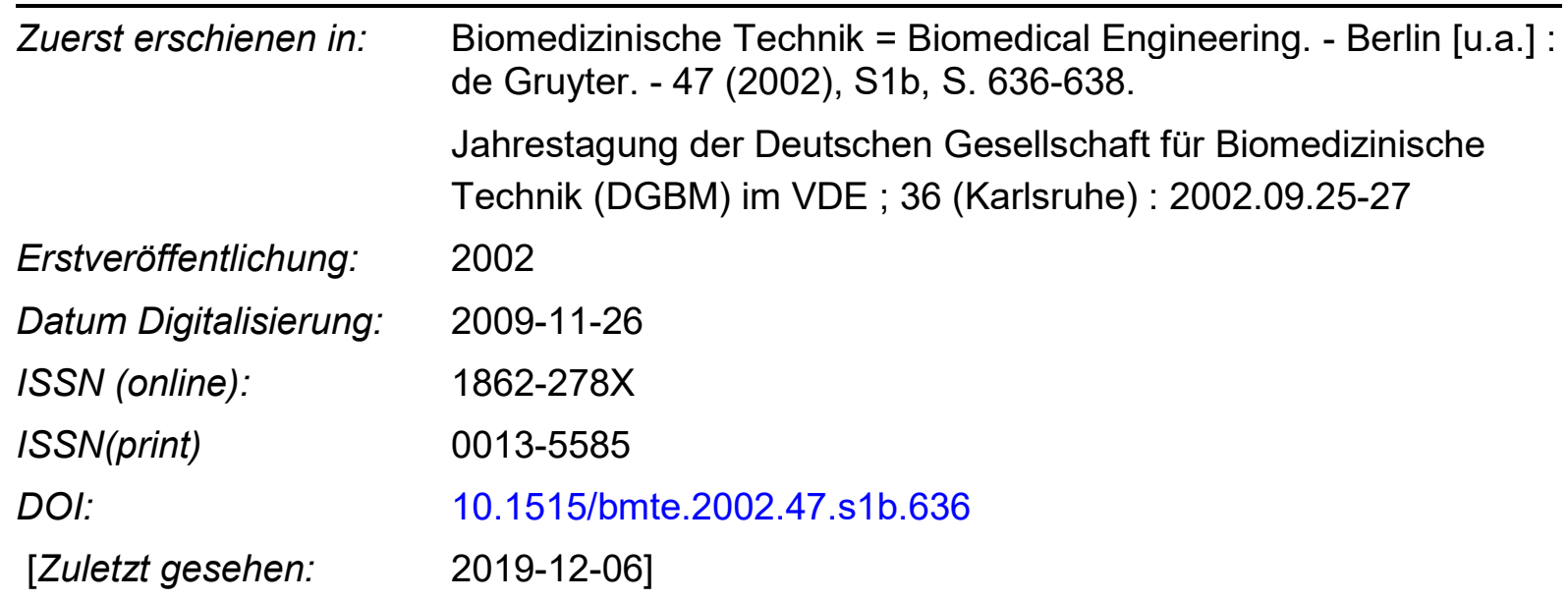

„Im Rahmen der hochschulweiten Open-Access-Strategie für die Zweitveröffentlichung identifiziert durch die Universitätsbibliothek IImenau."

"Within the academic Open Access Strategy identified for deposition by IImenau University Library."

„Dieser Beitrag ist mit Zustimmung des Rechteinhabers aufgrund einer (DFGgeförderten) Allianz- bzw. Nationallizenz frei zugänglich."

„This publication is with permission of the rights owner freely accessible due to an Alliance licence and a national licence (funded by the DFG, German DFG

Research Foundation) respectively." 


\title{
HOUGH-TRANSFORMATION ZUR BILDVERARBEITUNG BEI DER BLICKRICHTUNGSBESTIMMUNG
}

\author{
Nico Thomae, Kai-Uwe Plagwitz, Peter Husar, Günter Henning \\ Institut für Biomedizinische Technik und Informatik, Technische Universität Ilmenau, Deutschland
}

peter.husar@tu-ilmenau.de

\begin{abstract}
Die meisten Verfahren zur berührungslosen Blickrichtungsbestimmung sind kamerabasierend und werten das Bild des Auges aus. Häufig wird das Cornealreflexverfahren verwendet. Die Bildauswertung ist bei bisherigen Methoden stark störanfällig bei nicht geschlossenen Strukturen oder stark verrauschten Bildern. Ein robuster Ansatz bietet sich durch die HoughTransformation (HT) [1] des Kantenbildes in einen Parameterraum (PR). Nachteilig ist der immense Rechenaufwand, der eine Echtzeitanwendung der generalisierten $H T$ derzeit ausschließt. Als präferierte Lösung werden kombinierte Methoden für eine speziell angepasste HT diskutiert, die die Parameterzahl verringern, den Rechenaufwand senken und die Stabilität erhalten.
\end{abstract}

Keywords- eye-tracking, Blickrichtungsbestimmung, Bildverarbeitung, Hough-Transformation

\section{Einleitung}

Die Untersuchung zur Früherkennung und Einschätzung von Schädigungen der Netzhaut setzt bei konventionellen Verfahren eine aktive Mitarbeit des Untersuchten voraus. Dies bezieht sich auf die Fähigkeit und den Willen einen definierten Punkt im Gesichtsfeld zu fixieren und die Wahrnehmung eines Lichtreizes zu signalisieren. Sind diese Voraussetzungen nicht erfuilt, ist die diagnostische Aussagefähigkeit der Messung eingeschränkt und objektive Untersuchungsverfahren sind wünschenswert. Aus den bisherigen Vorarbeiten im Fachgebiet Biomedizinische Technik ist bekannt, dass die Fixation automatisch kontrollierbar ist und die Reizwahrnehmung anhand des EEG objektiv nachgewiesen werden kann. Die bei Patienten häufig vorkommenden Fehlfixationen sowie die Notwendigkeit wiederholter monofokaler Reizung führen jedoch zu Untersuchungszeiten, die in der klinischen Routine inakzeptabel sind. Zur Lösung dieser Probleme werden im Vorhaben folgende Ansätze verfolgt: Die Blickrichtung wird in Echtzeit ermittelt und das Reizmuster der aktuellen Blickrichtung nachgefuihrt. Dieser Beitrag wird sich speziell mit Methoden zur Ermittlung der aktuellen Blickrichtung auseinandersetzen.

\section{Methoden}

Zur Blickrichtungsbestimmung nach dem Corncalreflexverfahren ist es notwendig, dic Pupille als solches bzw. in diesem Fall deren Kontur aus dem Videobild au extrahieren. Dafür wird das urspringliche Grauwertbild zunächst einer histogrammgebundenen Schwellwertfunktion unterzogen. Anschließend wird mit einem Kantenoperator die Kontur der Pupille extrahiert. Aufgrund der limitierten Rechenzeit wird hier ein einfacher Sobeloperator verwendet. Danach kann mit Hilfe geeigneter Methoden der Mittelpunkt der Pupille bestimmt werden. Da bisherige Verfahren störanfällig gegenüber nicht geschlossenen Strukturen bzw. stark verrauschten Ursprungsbildern sind, wurde hier auf die HoughTransformation (HT) zurückgegriffen. Ist der Mittelpunkt der Pupille bestimmt, kann man mit Hilfe der Position des Comealreflexes und den Mittelpunktskoordinaten über einen vorher erstellten Kalibrationsdatensatz die aktuelle Blickrichtung bestimmen. Die genaue, schnelle und robuste Bestimmung des Pupillenmittelpunktes stellt dabei eindeutig die Hauptaufgabe dar. Die Standard HoughTransformation (SHT) läuft im wesentlichen nach folgenden 4 Punkten ab [2]:

1. die Menge $D$ aller Kantenpunkte im Binärbild bestimmen,

2. jeden dieser Punkte aus $D$ über eine Parametergleichung in den Parameterraum transformieren,

3. inkrementieren der durch die Parametergleichung beschriebenen Punkte in einem Akkumulatorfeld,

4. Detektion der lokalen Maxima in diesem Akkumulatorfeld, wobei jedes Maximum mit jeweils einer gesuchten Struktur im Ursprungsbild korrespondiert,

und ist geeignet einfache Strukturen wie Linien, Kreise und Ellipsen, über deren jeweilige Parametergleichung in einem Ursprungsbild aufzufinden. Eine Gerade lässt sich in der Form

$$
y=m x+n
$$

beschreiben. $(x, y)$ sind dabei die Koordinaten des Ursprungsbildes. Die dazugehörige Transformationsgleichung mit dem Parameterpaar $(n, m)$ würde dann folgendermaßen aussehen:

$$
n=-x m+y
$$

Wird das Verfahren auf Kreise enveitert, steigt natiirlich auch die Anzahl der Parameter. Ein Kueis lässt sich in der Form: 


$$
R^{2}=(x-a)^{2}-(y-b)^{2} \quad,(R>0)
$$

mit den Parametern $(R, a, b)$ beschreiben. Bci bekanntem Radius, und damit unter Konstanthaltung von $R$ ergeben sich folgende Transformationsgleichungen:

$$
b=y+\sqrt{R^{2}-(x-a)^{2}}
$$

und

$$
b=y-\sqrt{R^{2}-(x-a)^{2}}
$$

Ist $\mathrm{R}$ nicht bekannt und muss somit noch zusätzlich als Parameter bestimmt werden, vergrößert sich die Dimension des Parameterraumes von 2 auf 3. Unter der Annahme, dass sich die Pupillenkontur mit einer Ellipse beschreiben lässt, ergibt sich folgende Beschreibung [3]:

$a(x-p)^{2}+2 b(x-p)(y-q)+c(y-q)^{2}=1$

mit der Einschränkung:

$$
a c-b^{2}>0
$$

Da der entstehende Parameterraum damit fünfdimensional ist und sowohl die Rechenzeit als auch der Speicherbedarf mit jedem zusätzlichen Parameter expotential ansteigt, ist davon auszugehen, dass die SHT zwar eine Lösung des Problems darstellt, aber mit dem Stand der heutigen Rechentechnik nicht in Echtzeit durchführbar ist. Die SHT zeichnet sich aber, im Gegensatz zu anderen Verfahren, prinzipbedingt durch ihre große Robustheit gegenüber nichtgeschlossenen Konturen aus, welche natürlich jederzeit durch Artefakte im Ursprungsbild entstehen können.

\section{Geht man von folgenden Einschränkungen aus:}

1. die Pupillenkontur wird als Ellipse angenähert,

2. es werden nur die Mittelpunktskoordinaten benötigt,

3. und es existiert nur eine ellipsenförmige Struktur im Ursprungsbild,

so kann im folgenden gezeigt werden, wie nun die gewünschte Schnelligkeit bei gleichzeitiger Robustheit speziell für das vorliegende Problem erreicht werden kann. Unter anderem von Mc Laughlin [3] [4] wird beschrieben, wie das fünfdimensionale nichtlineare Parametersystem in teilweise lineare Teilsysteme zerlegt werden kann. So wird z.B. die Bestimmung des Mittelpunktes $(p, q)$ unter Ausnutzung spezieller geometrischer Eigenschaften einer Ellipse auf ein zweidimensionales, lineares Problem reduziert.

Wählt man, wie in Abb.1 gezeigt, drei Punkte P1,P2,P3 auf einer Ellipse aus, und legt in diesen Punkten jeweils die Tangenten an, kann aus dem Schnittpunkt der
Geraden $t / m I$ und $t 2 \mathrm{~m} 2$ den Mittelpunkt der Ellipse bestimmen. $m l, m 2$ sind dabei die Mittelpunkte der Verbindungslinien zwischen $P 1, P 2$ bzw. $P 1, P 3$ und $/, \imath 2$ die Schnittpunkte der jeweiligen Tangenten.

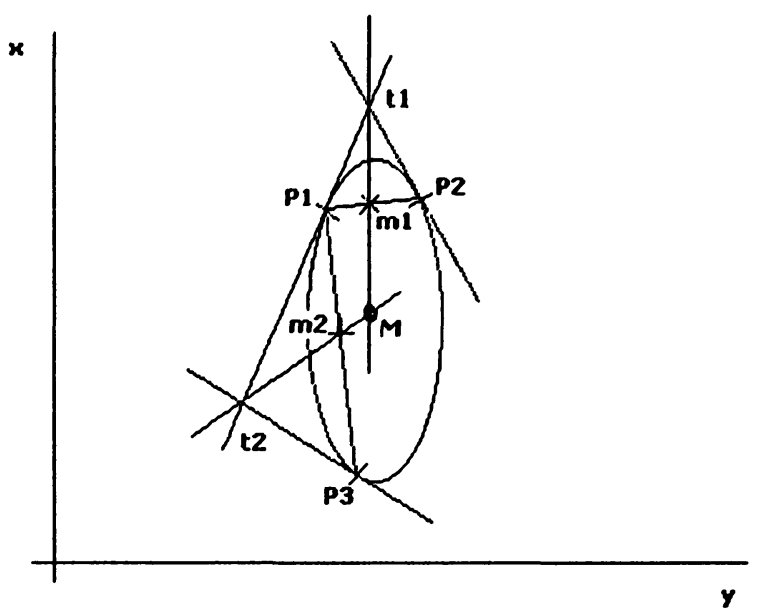

Abbildung 1: Mittelpunktsbestimmung

Bei der Auswahl der Punkte $P 1, P 2, P 3$ ist das PunktAbstand-Kriterium (PAK) [2] einzuhalten, damit die ausgewählten Punkte weder $\mathrm{zu}$ nahe noch $\mathrm{zu}$ weit voneinander entfernt liegen.

$$
\text { dist }_{\min } \leq \operatorname{dist}(P 1, P 2, P 3) \leq \text { dist }_{\max }
$$

Kombiniert man nun diese Mittelpunktsbestimmung mit einer speziellen HT, der sogenannten Random HoughTransformation (RHT), reduzieren sich die Rechenzeit und der Speicherbedarf im Gegensatz zur SHT für die Bestimmung des Mittelpunktes drastisch. Außerdem bleibt die Robustheit größtenteils erhalten.

Der Algorithmus der RHT für Ellipsen lässt sich mit folgenden 6 Punkten beschreiben [2]:

1. die Menge $\mathrm{D}$ aller Kantenpunkte im Binärbild bestimmen

2. 3 Punkte (P1,P2,P3) zufällig aus D auswählen

3. falls die Punkte das PAK nicht erfüllen nochmals Punkt 2. ausführen

4. Bestimmung des Mittelpunktes (p,q) nach o.g. Verfahren

5. die entsprechende Zelle (Pixel) in einem Akkumulatorfeld inkrementieren, wobei das Akkumulatorfeld den Parameterraum $P(p, q)$ repräsentiert

6. testen ob das entstehende Maximum im Akkumulatorfeld einen festgelegten Schwellwert überschritten hat, wenn ja, dann Abbruch, wenn nein, wieder zu 2. springen

\section{Ergebnisse}

Nach ersten Tests mit der Kombination beider Methoden, an realen Bildern lässt sich feststellen, dass die Genauigkeit 
des Tangentenfittings sich unmittelbar auf die Genauigkeit der Mittelpunktsbestimmung niederschlägt. Sichtbar wird dies durch die große Streuung der möglichen Mittelpunktsparameter $(p, q)$ im Parameterraum (Abb.2) Ziel ist es also, das Fitting der Tangenten zu verbessern, die Streuung deutlich zu reduzieren, und somit die Anzahl der Durchläufe und damit letztendlich die Rechenzeit weiter zu verringern.

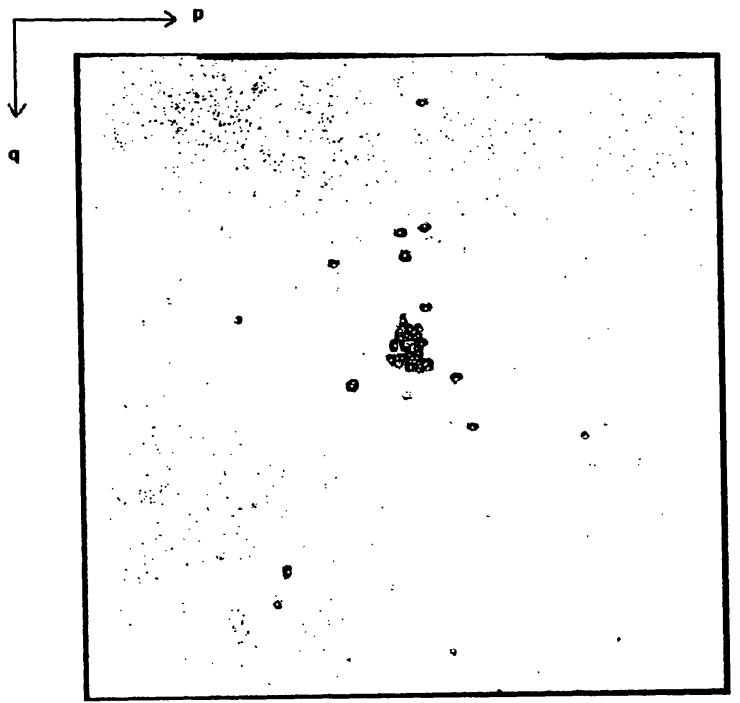

Abbildung 2: PR nach erfolgter Transformation

Trotz einiger Ausreißer wurde der Mittelpunkt sicher erkannt. (Abb. 3)

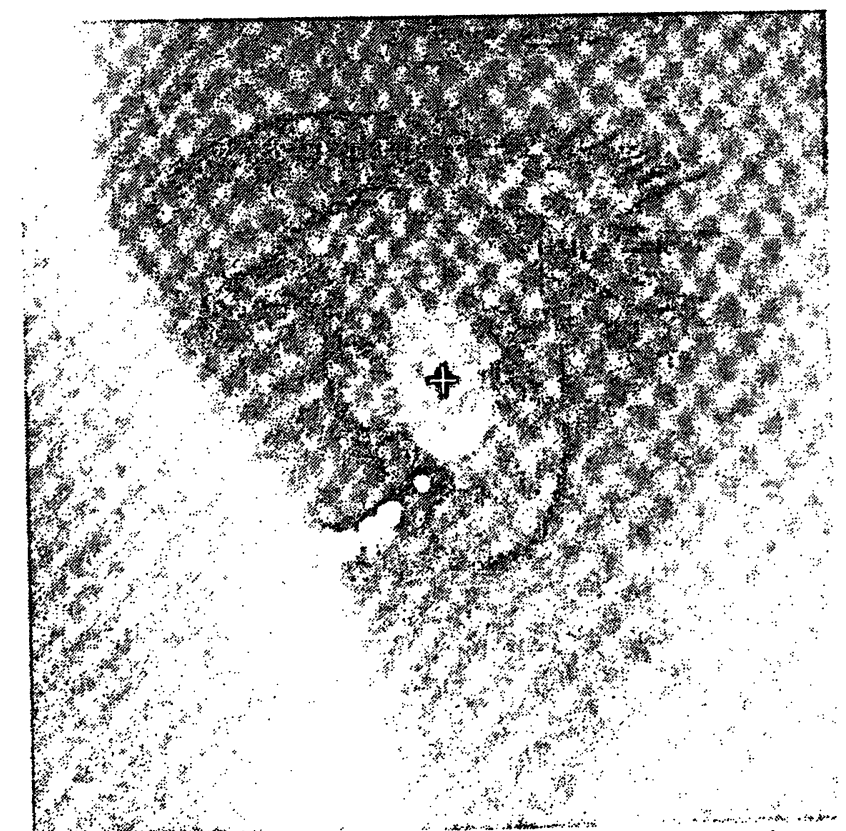

Abbildung 3: Markierung des errechneten Mittelpunktes

\section{Literaturverzeichnis}

[1] P.V.C Hough, Method and means for recognizing complex patterns. U.S. Patent 3,069,654,1962

[2] H. Kälviäinen, „Randomized Hough Transform - New Extensions" Research Papers pp. 3-4, 1994

[3] R.A. Mc Laughlin, „Randomized Hough Transform: Improve Ellipse Detection with Comparison" Technical Report ,1992

[4] H.K.Yuen, J. Illingworth, J. Kittler, „Detecting partially occluded ellipses using the Hough transform", Image and Vision Computing, 7, pp. 31-37, 1989 